\title{
MAN.IO - The review process of Bio-Manguinhos' strategic plan and its immediate impacts
}

Rosangela Vianna Alves da Silva ${ }^{1 *}$; Elaine Vasconcelos do Nascimento Gomes ${ }^{1}$; Lethícia Mallet Vivas $^{1}$; Monique Menegaci Barbosa ${ }^{1}$; Pedro Ribeiro Barbosa ${ }^{2}$.

1Fiocruz/Bio-Manguinhos;

2IBMP- Instituto de Biologia Molecular do Paraná.

Introduction: The Immunobiological Technology Institute (Bio-Manguinhos) has recently finalized its Strategic Plan for the next 10 years. The challenge of conducting it to a sustainable future is based on search for excellence in management, investment in the development of products, services and innovative processes, modernization of physical facilities, socioenvironmental responsibility, strengthening of technological platforms and essential skills to the consolidation of its strategic role for Public Health. At the current Brazilian political juncture, short-term scenarios require immediate actions, demanding an option of fast organizational mobilization, by differentiated actions for the short-term - 2019-2020 - while taking into account the medium and long-term scenarios.

Objective: Present the case study of Bio-Manguinhos strategic plan review, assuming the horizon of 2028 for the long-term process, at the same time that establishes an initial period of two years - 2019-2028 - for consolidation operations of the recent organizational achievements, and the implementation of new conditions for the long-term development.

Methodology: The review of Bio-Manguinhos Strategic Plan assumed the adoption of a participatory process. In a one-year work it, involved hundreds of managers, collaborators and specialists, internal and external, in 5 workshops and dozens of meetings that counted with the participation of practically all areas of the Institute. As intermediate products, we can cite the work of prospecting trends of the external environment, the elaboration of the internal environment's diagnosis and the design of a tool for mapping and monitoring Bio-Manguinhos' stakeholders. Besides the SWOT Matrix, there were also developed technological roadmaps by product segment - vaccines, biopharmaceuticals and diagnosis - as well as proposal of management model of technological development. The consolidated work was the basis for the elaboration of the strategic direction statement for the next 10 years.

Results: The institutional priorities for the next 10 years were translated into in corporate strategies grouped into 4 strategic axes - innovation, efficiency, compliance and organizational intelligence - which were deployed in three strategies of market positioning and three of technological positioning. At the tactical level, they are reflected in the 25 institutional drivers and 28 business drivers grouped by product segment. At the operational level, they are translated into the set of strategic portfolio - projects and initiatives - that execute the idealized planning. The design of the plan's management and governance proposal is in progress.

Conclusion: Clear drivers for the establishment of operational priorities were the main gain of the strategic plan review. Although it is facilitated by the participatory process, the materialization of the current strategic agenda will require the construction of an institutional performance monitoring system that facilitates the integration of planning with the operation and is able to signal the need for adjustments and/or technological and market redirects, whenever necessary.

Keywords: Strategic Management; Strategy; Planning 